\title{
Identification of growth seeds in the neonate brain through surfacic Helmholtz decomposition
}

\author{
Julien Lefèvre ${ }^{1}$, François Leroy ${ }^{1}$, Sheraz Khan ${ }^{2}$, Jessica Dubois ${ }^{1}$, Petra S. \\ Huppi $^{3}$, Sylvain Baillet ${ }^{2}$, and Jean-François Mangin ${ }^{1}$ \\ 1 Neurospin, CEA, Saclay, France \\ \{julien.lefevre, jean-francois.mangin\}@cea.fr \\ 2 Cognitive Neuroscience and Brain Imaging Laboratory, \\ CNRS UPR640-LENA, Université Pierre et Marie CuRIE-Paris6, Paris, France \\ 3 Department of Pediatrics, Geneva University Hospitals 1211, Geneva 4, Switzerland
}

\begin{abstract}
We report on a new framework to investigate the rapid brain development of newborns. It is based on the analysis of depth maps of the cortical surface through the study of a displacement field estimated by surfacic optical flow methods. This displacement field show local evolution of sulci directly on the cortical surface. Detection of its critical points is performed with the Helmholtz decomposition which allow to identify sources of the developmental process. They can be viewed as growth seeds or in other terms points around which the sulcal growth organizes itself. We show the reproducibility of such growth seeds across 4 neonates and make a link of this new concept to the "sulcal roots" one proposed to explain the variability of human brain anatomy.
\end{abstract}

\section{Introduction}

Recent studies in MRI have described precisely the ontogenesis of the cortical folding during early phases of development [5],[17]. Thank to these studies it becomes now possible to follow the evolution of brain structures during the gyrification, that is to say to detect potential lesions [5] or to understand step by step the complex processes of sulci formation whose physiological basis are yet not well deciphered [14] [15] [16]. Through postprocessing tools [5] the authors extracted the surface between gray and white matter of preterm newborns at critical ages (26-36 weeks). In [18] a registration algorithm is presented in order to align cortical surfaces in longitudinal studies and to track the cortical development. This tracking has been evaluated on sulci which are 3D structures and very few methods have been previously performed directly on the surfaces. In [3] a primal sketch of the cortical mean curvature allows to detect elementary cortical folds and fold merging during brain development.

However in the study of functional brain activations a recent work [10] has shown the possibility to follow evolving texture - MEG neural activities in the present case - directly on the cortical surfaces through optical flow algorithms. Moreover the knowledge of such a deformation field allows to detect local patterns of growth in particular focal point around which the growth spreads [6]. 
This last study gives future possibilities for validating model of cortical growth such as the "sulcal roots" model in which atoms of future sulci are supposed to preexist in the developing embryo [13].

The aim of our study is to propose a framework which allows to track the changes in the sulcation of neonates along time and directly on the cortical surface. The advantage of working on surfaces is twofold since there is a gain in computation time with respect to $3 \mathrm{D}$ analysis and we offer a practical visualization of displacement fields.

We will define a quantity of interest which is based on depth maps applied projected to cortical surfaces. Then using non linear registration technique based on iconic feature [4] we will make correspond brain surfaces of neonates and their depth maps longitudinally. Next we apply a recent methodology to track evolutions of scalar measures on surfaces through generalized optical flow [9]. At last we perform a helmholtz decomposition of the resulting deformation field that will allow to identify in it robust features such as sources of growth. By this way we will demonstrate that the evolution field of the developing brain has a radial structure and organizes itself into growth seeds.

We evaluate the reproducibility of these growth seeds using a rigid registration of the cortical surfaces and show that we find some clusters of seeds that can be compared to anatomical features ("sulcal roots") previously described in the literature.

\section{Optical flow on surfaces}

In this part we recall the formalism introduced in [9] based on differential geometry to extend the computation of optical flow equation on Riemannian manifolds. This theoretical development will allow to deduce a vector field that represents the evolution of a scalar quantity defined on a surface (typically the depth maps in the next application) along time.

\section{$2.1 \quad$ Notations}

Here are some notations : $\mathcal{M}$ is a 2-Riemannian manifold representing the imaging support (i.e. the cortical surfaces) and $I(p, t)$ a scalar quantity defined on a 2 -dimensional surface and in time. We note $\mathbf{e}_{\alpha}=\partial_{x_{\alpha}} p$, the canonical basis with respect to a coordinate system $x_{\alpha}$ of the tangent space $T_{p} \mathcal{M}$ at a point $p$ of the manifold, and $T \mathcal{M}=\bigcup_{p} T_{p} \mathcal{M}$ the tangent bundle of $\mathcal{M}$.

$\mathcal{M}$ is equipped with a Riemannian metric, that is there exists a positivedefinite form: $g_{p}: T_{p} \mathcal{M} \times T_{p} \mathcal{M} \rightarrow \mathbb{R}$. A natural choice for $g_{p}$ is the restriction of the Euclidian metric to $T_{p} \mathcal{M}$, which we have adopted for subsequent computations. For concision purposes, we will now only refer to $g_{p}$ as $g$.

The classical hypotheses for computing optical flow [8] leads to the equation:

$$
\partial_{t} I+g\left(\mathbf{V}, \nabla_{\mathcal{M}} I\right)=0 .
$$


Since only the component of the flow $\mathbf{V}$ in the direction of the gradient is accessible to estimation (aperture problem) [8], additional constraints on the flow are needed to yield a unique solution. This approach classically reduces to minimizing an energy functional such as in [8]:

$$
\mathcal{E}(\mathbf{V})=\int_{\mathcal{M}}\left(\frac{\partial I}{\partial t}+g\left(\mathbf{V}, \nabla_{\mathcal{M}} I\right)\right)^{2} \mathrm{~d} \mu+\lambda \int_{\mathcal{M}} \mathcal{C}(\mathbf{V}) \mathrm{d} \mu,
$$

The first term is a measure of fit of the optical flow model to the data, while the second one acts as a spatial regularizer of the flow. The smoothness term can be expressed as a Frobenius norm:

$$
\mathcal{C}(\mathbf{V})=\operatorname{Tr}\left({ }^{t} \nabla \mathbf{V} \cdot \nabla \mathbf{V}\right) \text { and }(\nabla \mathbf{V})_{\alpha}^{\beta}=\partial_{\alpha} V^{\beta}+\sum_{\gamma} \Gamma_{\alpha \gamma}^{\beta} V^{\gamma}
$$

$(\nabla \mathbf{V})$ is the covariant derivative of $\mathbf{V}$, a generalization of vectorial gradient. $\partial_{\alpha} V^{\beta}$ is the classical Euclidian expression of the gradient, and $\sum_{\gamma} \Gamma_{\alpha \gamma}^{\beta} V^{\gamma}$ reflects local deformations of the tangent space basis since the Christoffel symbols $\Gamma_{\alpha \gamma}^{\beta}$ are the coordinates of $\partial_{\beta} \mathbf{e}_{\alpha}$ along $\mathbf{e}_{\gamma}$. This expression ensures the tensoriality property of $\mathbf{V}$, i.e. the invariance with parametrization changes.

\subsection{Variational formulation}

Deriving a variational formulation from the minimization of (2) ensures the wellposedness of the problem - existence and unicity of the solution in a specific and convenient function space - and allows to solve numerically the problem on discrete irregular surfacic tessellations.

$\Gamma^{1}(\mathcal{M})$ is the working space of vector fields on which functional $\mathcal{E}(\mathbf{V})$ will be minimized. We chose a space of vector fields in which the coordinates of each element are located in $C^{1}(\mathcal{M})$ (the space of differentiable functions on the manifold):

$$
\Gamma^{1}(\mathcal{M})=\left\{\mathbf{V}: \mathcal{M} \rightarrow T \mathcal{M} / \mathbf{V}=\sum_{\alpha=1}^{2} V^{\alpha} \mathbf{e}_{\alpha}, V^{\alpha} \in C^{1}(\mathcal{M})\right\}
$$

with the scalar product :

$$
<\mathbf{U}, \mathbf{V}>_{\Gamma^{1}(\mathcal{M})}=\int_{\mathcal{M}} g(\mathbf{U}, \mathbf{V}) \mathrm{d} \mu+\int_{\mathcal{M}} \operatorname{Tr}\left({ }^{t} \nabla \mathbf{U} \nabla \mathbf{V}\right) \mathrm{d} \mu .
$$

$\mathcal{E}(\mathbf{V})$ can be simplified from (2) as a combination of a constant $K(t)$, a linear and a bilinear form :

$$
\begin{aligned}
f(\mathbf{U}) & =-\int_{\mathcal{M}} g\left(\mathbf{U}, \nabla_{\mathcal{M}} I\right) \partial_{t} I \mathrm{~d} \mu \\
a(\mathbf{U}, \mathbf{V}) & =\int_{\mathcal{M}} g\left(\mathbf{U}, \nabla_{\mathcal{M}} I\right) g\left(\mathbf{V}, \nabla_{\mathcal{M}} I\right) \mathrm{d} \mu+\lambda \int_{\mathcal{M}} \operatorname{Tr}\left({ }^{t} \nabla \mathbf{U} \nabla \mathbf{V}\right) \mathrm{d} \mu .
\end{aligned}
$$


Minimizing $\mathcal{E}(\mathbf{V})$ on $\Gamma^{1}(\mathcal{M})$ is then equivalent to the following problem :

$$
\min _{\mathbf{V} \in \Gamma^{1}(\mathcal{M})}(a(\mathbf{V}, \mathbf{V})-2 f(\mathbf{V})+K(t))
$$

It has been proved in [9] that there exists a unique solution. Moreover, this solution $\mathbf{V}$ to (4) satisfies:

$$
a(\mathbf{V}, \mathbf{U})=f(\mathbf{U}), \forall \mathbf{U} \in \Gamma^{1}(\mathcal{M})
$$

\section{Helmholtz decomposition of a vector field}

Helmholtz decomposition is a classical way to decompose a vector field into the sum of a rotational part and a divergential part as illustrated on Fig.1. This technique allows to detect the singularities of a vector field, that is source, sink or rotation center [12]. It has been recently used [7] in cardiac video analysis in order to track the critical points in the heart which can lead to a better understanding of the dynamics of the cardiac electrical activity and its anomalies. Identification of such points for a brain growth field seems to be of highest importance to characterize the underlying spatiotemporal evolution.

More formally we have the following theorem :

Theorem : Given $\mathbf{V}$ a vector field in $\Gamma^{1}(\mathcal{M})$, there exists unique functions $U$ and $A$ in $L^{2}(\mathcal{M})$ and a vector field $\mathbf{H}$ in $\Gamma^{1}(\mathcal{M})$ such as :

$$
\begin{aligned}
\mathbf{V} & =\nabla_{\mathcal{M}} U+\mathbf{C u r l}_{\mathcal{M}} A+\mathbf{H} \\
\operatorname{div}_{\mathcal{M}} \mathbf{H} & =0 \quad \operatorname{curl}_{\mathcal{M}} \mathbf{H}=0
\end{aligned}
$$

Notations : In our applications $\mathcal{M}$ is a surface (or submanifold) thus it is possible to get a normal vector in each point :

$$
\mathbf{n}_{p}=\frac{\partial}{\partial x_{1}} \wedge \frac{\partial}{\partial x_{2}}
$$

The normal does not depend on the choice of the parametrization $\left(x_{1}, x_{2}\right)$. Then we define the divergence operator through duality :

$$
\int_{\mathcal{M}} U \operatorname{div}_{\mathcal{M}} \mathbf{H}=-\int_{\mathcal{M}} g\left(\mathbf{H}, \nabla_{\mathcal{M}} U\right)
$$

Scalar and vectorial curl are at last :

$$
\operatorname{Curl}_{\mathcal{M}} A=\nabla_{\mathcal{M}} A \wedge \mathbf{n} \quad \operatorname{curl}_{\mathcal{M}} \mathbf{H}=\operatorname{div}_{\mathcal{M}}(\mathbf{H} \wedge \mathbf{n})
$$

With these formulas we have intrinsic expressions which do not depend on the parametrization of the surface. 

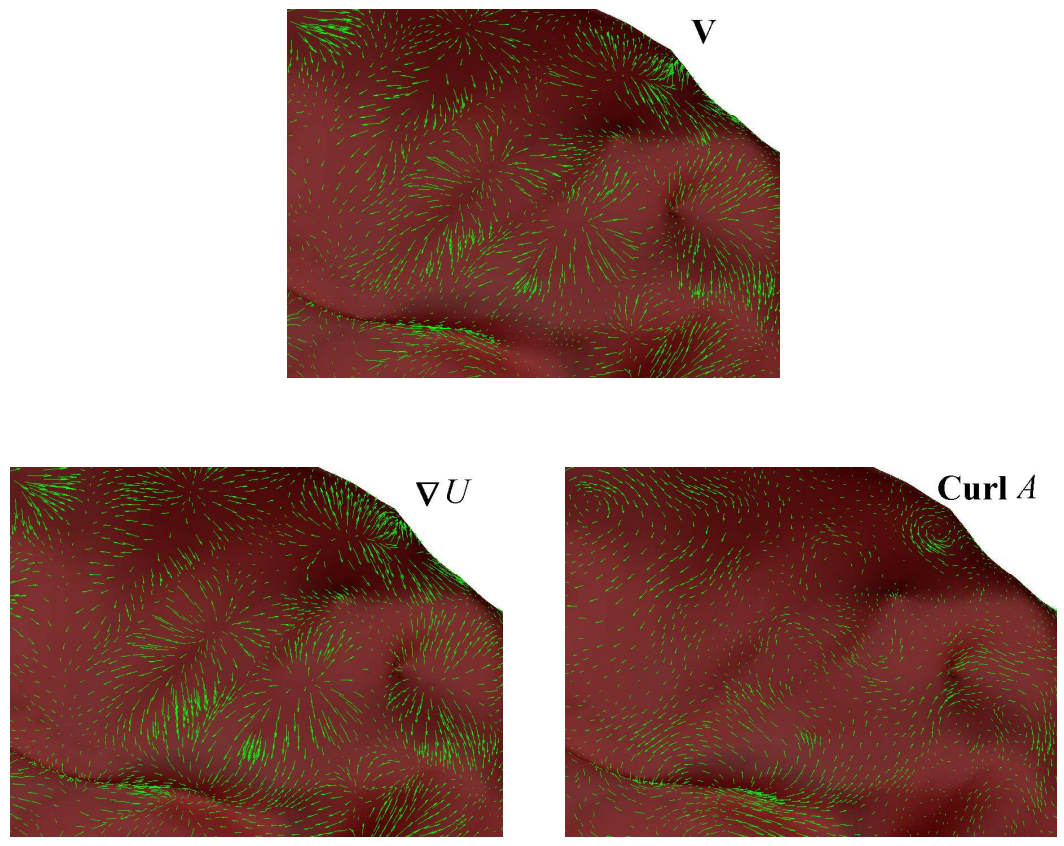

Fig. 1. First line : Vector field on a cortical mesh. Second line : divergential and rotational parts of the vector field. We can note that the vector field is mainly divergential and we can identify visually that it has only sources and no sinks.

Proof: The proof of the existence of a solution follows a classical construction. It can be shown that if $U$ and $A$ minimize the two functionals :

$$
\begin{gathered}
\int_{\mathcal{M}}\left\|\mathbf{V}-\nabla_{\mathcal{M}} U\right\|^{2} \\
\int_{\mathcal{M}}\left\|\mathbf{V}-\operatorname{Curl}_{\mathcal{M}} A\right\|^{2}
\end{gathered}
$$

$\mathbf{V}-\nabla_{\mathcal{M}} U-\operatorname{Curl}_{\mathcal{M}} A$ is solution of (7).

These two previous functionals are convex therefore they have unique minimum on $L^{2}(\mathcal{M})$ which satisfies :

$$
\begin{aligned}
\forall \phi \in L^{2}(\mathcal{M}), \int_{\mathcal{M}} g\left(\mathbf{V}, \nabla_{\mathcal{M}} \phi\right) & =\int_{\mathcal{M}} g\left(\nabla_{\mathcal{M}} U, \nabla_{\mathcal{M}} \phi\right) \\
\forall \phi \in L^{2}(\mathcal{M}), \int_{\mathcal{M}} g\left(\mathbf{V}, \mathbf{C u r l}_{\mathcal{M}} \phi\right) & =\int_{\mathcal{M}} g\left(\mathbf{C u r l}_{\mathcal{M}} A, \mathbf{C u r l}_{\mathcal{M}} \phi\right)
\end{aligned}
$$




\section{Numerical aspects}

Once proved the well-posedness of the regularized optical flow problem and the Helmholtz decomposition, we derive numerical methods from the variational formulations (5), (8) and (9).

Optical flow : First we consider the vector space of continuous piecewise affine vector fields which belong to the tangent space at each node of a mesh $\widehat{\mathcal{M}}$. A basis is:

$$
\mathbf{W}_{\alpha, i}=w(i) \mathbf{e}_{\alpha}(i) \text { for } 1 \leq i \leq \operatorname{Card}(\widehat{\mathcal{M}}), \alpha \in\{1,2\},
$$

where $w(i)$ stands for the continuous piecewise affine function which is 1 at node $i$ and 0 at all other triangle nodes, and $\mathbf{e}_{\alpha}(i)$ is a basis of tangent space at node $i$.

The variational formulation in (5) yields the linear system:

$$
\left[a\left(\mathbf{W}_{\alpha, i}, \mathbf{W}_{\beta, j}\right)\right]_{(\alpha, i),(\beta, j)}[\mathbf{V}]=\left[f\left(\mathbf{W}_{\alpha, i}\right)\right]_{\alpha, i}
$$

where $[\mathbf{V}]$ are the components of the velocity field $\mathbf{V}$ in the basis $\mathbf{W}_{\alpha, i}$. The matricial coefficient $a\left(\mathbf{W}_{\alpha, i}, \mathbf{W}_{\beta, j}\right)$ and $f\left(\mathbf{W}_{\alpha, i}\right)$ can be explicitly computed with first-order finite elements by estimating the integrals on each triangle $T$ of the mesh and summing the different contributions.

$\nabla_{\widehat{\mathcal{M}}} I$ is obtained on each triangle $T=[i, j, k]$ from the linear interpolation:

$$
\nabla_{\widehat{\mathcal{M}}} I=I(i) \nabla_{T} w(i)+I(j) \nabla_{T} w(j)+I(k) \nabla_{T} w(k) .
$$

with

$$
\nabla_{T} w(i)=\frac{\mathbf{h}_{\mathbf{i}}}{\left\|\mathbf{h}_{\mathbf{i}}\right\|^{2}},
$$

where $\mathbf{h}_{\mathbf{i}}$ is the height of triangle $T$ from vertex $i$.

Helmholtz decomposition : Equations 8) and (9) hold when we replace $\phi$ by the basis function $w_{i}$ so we have the two systems :

$$
\begin{aligned}
{\left[\int_{\widehat{\mathcal{M}}} g\left(\nabla_{\mathcal{M}} w(i), \nabla_{\mathcal{M}} w(j)\right)\right]_{i, j}[\mathbf{U}] } & =\left[\int_{\widehat{\mathcal{M}}} g\left(\mathbf{V}, \nabla_{\mathcal{M}} w(i)\right)\right]_{i} \\
{\left[\int_{\widehat{\mathcal{M}}} g\left(\mathbf{C u r l}_{\mathcal{M}} w(i), \mathbf{C u r l}_{\mathcal{M}} w(j)\right)\right]_{i, j}[\mathbf{A}] } & =\left[\int_{\widehat{\mathcal{M}}} g\left(\mathbf{V}, \mathbf{C u r l}_{\mathcal{M}} w(i)\right)\right]_{i}(12)
\end{aligned}
$$

Similarly to (10) we can compute each coefficient of the matrix on each triangle. So the two previous equations have the following expressions :

$$
\begin{aligned}
\sum_{T \ni i, j} \frac{\mathbf{h}_{\mathbf{i}}}{\left\|\mathbf{h}_{\mathbf{i}}\right\|^{2}} \cdot \frac{\mathbf{h}_{\mathbf{j}}}{\left\|\mathbf{h}_{\mathbf{j}}\right\|^{2}} \mathcal{A}(T) & =\sum_{T \ni i} \mathcal{A}(T) \mathbf{V} \cdot \frac{\mathbf{h}_{\mathbf{i}}}{\left\|\mathbf{h}_{\mathbf{i}}\right\|^{2}} \\
\sum_{T \ni i, j}\left(\frac{\mathbf{h}_{\mathbf{i}}}{\left\|\mathbf{h}_{\mathbf{i}}\right\|^{2}} \wedge \mathbf{n}\right) \cdot\left(\frac{\mathbf{h}_{\mathbf{j}}}{\left\|\mathbf{h}_{\mathbf{j}}\right\|^{2}} \wedge \mathbf{n}\right) \mathcal{A}(T) & =\sum_{T \ni i} \mathcal{A}(T) \mathbf{V} \cdot\left(\frac{\mathbf{h}_{\mathbf{i}}}{\left\|\mathbf{h}_{\mathbf{i}}\right\|^{2}} \wedge \mathbf{n}\right)
\end{aligned}
$$




\section{Application to the growth of the neonatal brain}

\subsection{Preprocessing}

Acquisition The dataset consists of 4 healthy infants. MR scans were acquired two times with T2 weighted sequence on a $3 \mathrm{~T}$ MRI system. The first acquisition was respectively, $0.6,0.7,1.4$ and -0.7 weeks with respect to 40 weeks of gestational age and the second one, 3.2, 4.6, 3.1 and 2 weeks. Slice resolution was 0.7 $\times 0.75 \times 0.7 \mathrm{~mm}$ for subjects $1,3,4$ and $0.78 \times 0.6 \times 0.78 \mathrm{~mm}$ for subject 2 .

Brain segmentation The segmentation of white and gray matter in neonate MRI is a challenging issue because of the inversion of contrast regarding adult MRI. We have used a dedicated algorithm to overcome this problem [11]. It is based on a characterization of tissue using features based on geometrical properties and the evolution of two surfaces located on each side of the GM-WM interface.

Depth maps Once the cortical surfaces have been segmented we use a specific treatment of BrainVisa [1] to compute their depth maps. They are obtained from the geodesic distance of the surface to a binary mask of the brain that has been dilated and eroded (respectively 5 and 2 voxels). Fig. 2 illustrates the resulting depth maps for two cortical surfaces of the same neonate taken at two different ages (birth, birth +4 weeks).
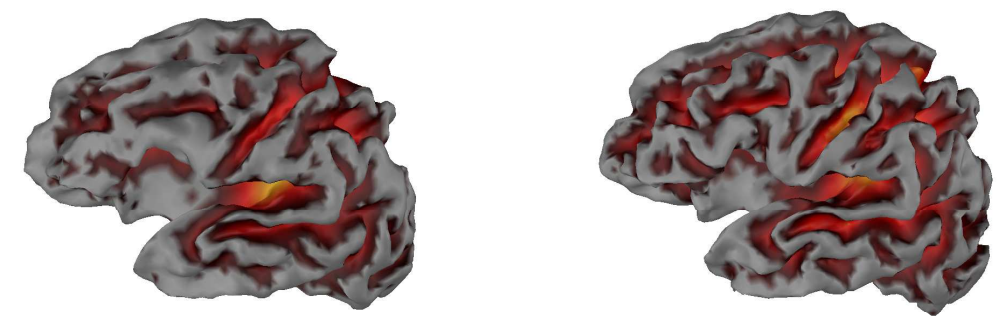

Fig. 2. Depth maps of two surfaces of the same subject at two different ages (birth, birth +4 weeks).

\subsection{Registration of cortical surfaces}

We have used two kinds of registration techniques.

First We have used iconic based non rigid registration [4] to make correspond the brain surfaces of each subject longitudinally. This method estimates a transformation $T$ between two 3D images $I$ and $J$ that must minimize iteratively the following energy : 


$$
S(I, J, C)+\sigma\|C-T\|^{2}+\lambda R(T)
$$

where $R$ is a quadratic regularization energy, $T$ an intermediate transformation and $\sigma, \lambda$ are parameters. The resulting volumic transformation is then applied to cortical surfaces.

After having registered the less mature cortical surface on the more mature one we interpolate the depth maps by a nearest neighbors method. So we have two depth maps at different time steps projected on the same surface. It becomes now possible to track the evolution of this map from a time step to another.

Secondly we have used a coarser method to make correspond the growth seeds across the subjects. It is based on the iterative closest point (ICP) algorithm [2] in order to register rigidly one surface onto another one chosen as a template. The initialization of the algorithm is done by making correspond the principal directions of the two surfaces. We use also a nearest neighbors projection to transform the depth maps.

\subsection{Identification of growth seeds}

Optical flow computation For visualization purposes we compute the optical flow between the two depth maps on a smooth version of the more mature cortical surface. On Fig. 3 we display the result of the computation in green and the depth map of the less mature cortical surfaces for the subject 1.

We can see the radial structure of the vector field which has suggested the use of the Helmholtz decomposition in order to locate points of big divergence.

Helmholtz decomposition We compute the two scalar potentials $U$ and $A$ involved in the Helmholtz decomposition. On Fig 4 we show only the divergential part of the field which is simply given by $\nabla U$. We elicit also the local minima of the potential $U$ which corresponds to sources of the optical flow.

Such sources correspond to locations in the brain around which the depth maps tend to grow.

Evaluation

- First we analyze the relative contribution of the divergential and rotational parts to the displacement field. We can see on Fig 5 the histograms of the norms of the divergential and rotational parts for one subject. It is interesting to compute the ratio of the norms to get the proportion of points where the divergential part is greater than the rotational part. We give the mean of this ratio for each subject on table 1. It confirms that the displacement field is mainly divergential and justify to consider only the local extrema of the potential function $U$.

- On Fig 6 we display the superposition of seeds computed for four subjects and rigidly registered on one of the four surfaces. For a better visualization we only show clusters of seeds that belong to spheres of a given radius. We 


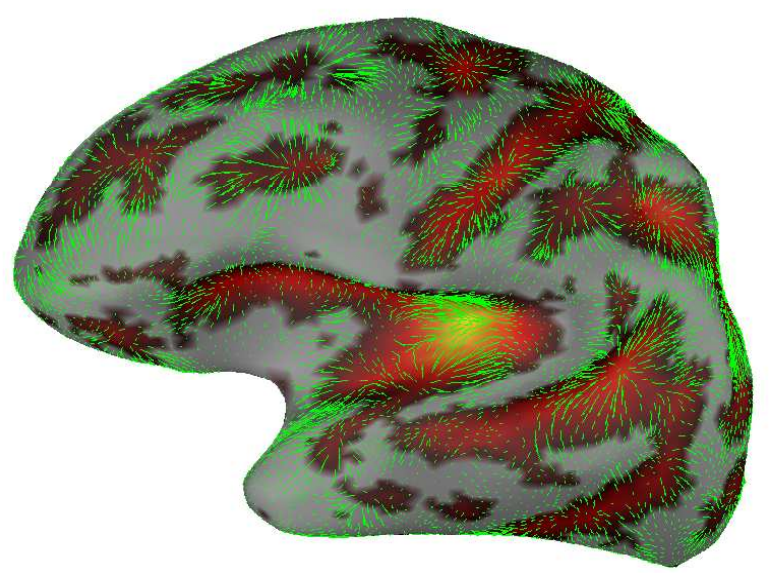

Fig. 3. Optical flow (green) on the smooth surface of the second time step and Depth maps of the first time step.

\begin{tabular}{|c||c|c|c|c|}
\hline$S_{1}$ & $S_{2}$ & $S_{3}$ & $S_{4}$ & \\
\hline Left hemisphere & $0.75 \pm 0.89$ & $0.13 \pm 0.88$ & $0.58 \pm 0.93$ & $0.76 \pm 0.95$ \\
\hline Right hemisphere & $0.61 \pm 0.93$ & $0.34 \pm 0.91$ & $0.35 \pm 0.96$ & $0.71 \pm 0.9$ \\
\hline
\end{tabular}

Table 1. Ratio of divergential and rotational norms (Log) for 4 subjects

also represent an average depth maps defined as the mean of the four depth maps after the nearest neighbors interpolation.

We can observe that despite the variability we can isolate a certain number of clusters (inside spheres). They can be compared to sulcal regions previously defined in the litterature [13] and called sulcal roots, anatomical structures in the sulcus fundi that are supposed to be strong reproducible landmarks. We suggest the following classification based on [13] : $1:$ Centralis superior, 2 : Centralis inferior, 3 : Frontallis superior posterior or Precentralis superior, 4 : fissura intraparietalis occipitalis superior, 5 : Frontalis inferior posterior or Precentralis inferior, 6 : Fronto-orbitalis, 7 : Temporal superior posterior, 8 : Temporal inferior posterior or Temporal inferior ascendens, 9 : Frontalis inferior anterior. 


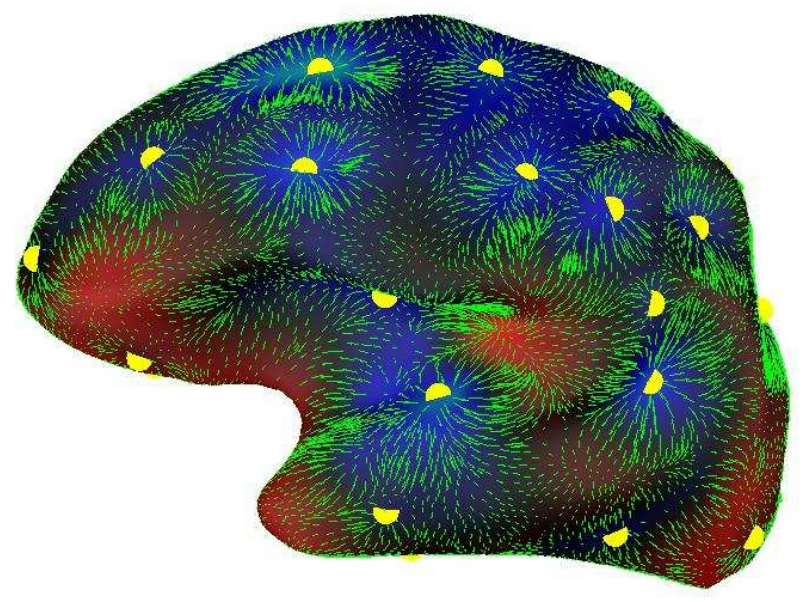

Fig. 4. Divergential part of the optical flow (green), potential map and local minima of $U$ in yellow.

\section{Conclusion}

We have reintroduced the concept of Helmholtz decomposition on manifolds. We have seen how, from a vector field, to extract potential functions that represent sources, sinks and rotation centers. We have applied this methodology to the displacement field computed from the evolution of depth maps of neonatal brains between the birth and four weeks later. We have shown that the displacement field organizes itself into growth centers of growth seeds that offer a certain reproducibility across four subjects. In future developments we propose to use a bigger cohort to yield statistic tests and comparisons between different classes of subjects and to identify disorders in the brain development. Moreover we plan to apply our method for aging where we could expect to detect sinks rather than sources in the evolution of depth maps.

\section{References}

[1] http://brainvisa.info/. 

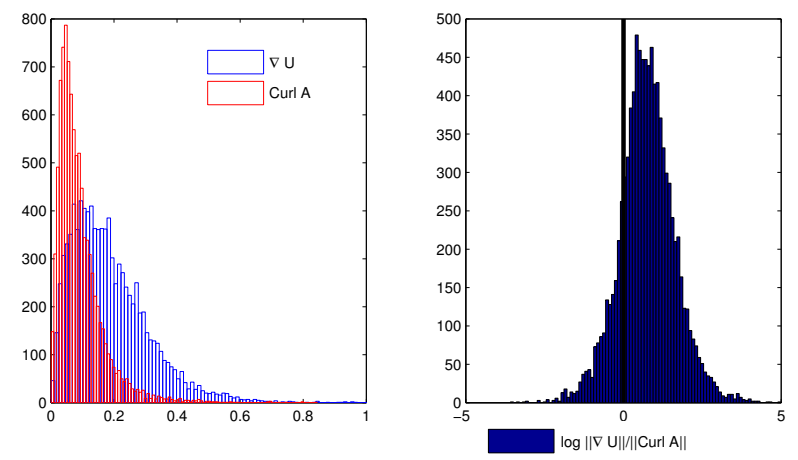

Fig. 5. Left: two histograms showing the norms of divergential part (blue) and rotational part (red). Right: Log ratio of the norms.

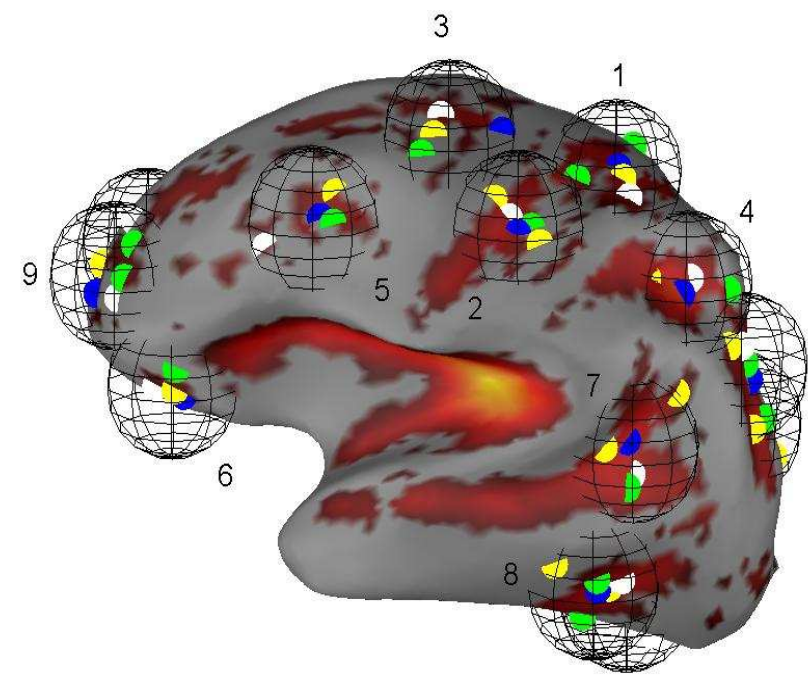

Fig. 6. Superposition of seeds for four subjects after affine registration and clustering. See text for legend. 
[2] P.J. Besl and N.D. McKay. A method for registration of 3-d shapes. IEEE Transactions on Pattern Analysis and Machine Intelligence, 14(2):239-256, 1992.

[3] A. Cachia, J.F. Mangin, D. Riviere, F. Kherif, N. Boddaert, A. Andrade, D. Papadopoulos-Orfanos, J.B. Poline, I. Bloch, M. Zilbovicius, P. Sonigo, F. Brunelle, and J. Regis. A primal sketch of the cortex mean curvature: a morphogenesis based approach to study the variability of the folding patterns. IEEE Trans. Medical Imaging, 22(6):754-765, 2003.

[4] P. Cachier, E. Bardinet, D. Dormont, X. Pennec, and N. Ayache. Iconic feature based registration: the pasha algorithm. Computer Vision and Image Understanding, 89(2-3):272-298, 2003.

[5] J. Dubois, M. Benders, A. Cachia, F. Lazeyras, R. Ha-Vinh Leuchter, SV Sizonenko, C. Borradori-Tolsa, JF Mangin, and PS Huppi. Mapping the Early Cortical Folding Process in the Preterm Newborn Brain. Cerebral Cortex, 2007.

[6] U. Grenander, A. Srivastava, and S. Saini. A Pattern-Theoretic Characterization of Biological Growth. Medical Imaging, IEEE Transactions on, 26(5):648-659, 2007.

[7] Q. Guo, M.K. Mandal, G. Liu, and K.M. Kavanagh. Cardiac video analysis using Hodge-Helmholtz field decomposition. Computers in Biology and Medicine, 36(1):1-20, 2006.

[8] B.K.P. Horn and B.G. Schunck. Determining optical flow. Artificial Intelligence, 17:185-204, 1981.

[9] J. Lefèvre and S. Baillet. Optical Flow and Advection on 2-Riemannian Manifolds: A Common Framework. IEEE Transactions on pattern analysis and machine intelligence, pages 1081-1092, 2008.

[10] J. Lefèvre, G. Obozinski, and S. Baillet. Imaging brain activation streams from optical flow computation on 2-riemannian manifold. Proc. Information Processing in Medical Imaging, pages 470-481, 2007.

[11] F. Leroy, J.F. Mangin, F. Rousseau, H. Glasel, L. Hertz-Pannier, J. Dubois, and G. Dehaene-Lambertz. Cortical Surface Segmentation in Infants by Coupled Surfaces Deformation across Feature Field. Imaging the Early Developing Brain: Challenges and Potential Impact, Workshop at MICCAI, 2008.

[12] K. Polthier and E. Preuss. Identifying vector fields singularities using a discrete hodge decomposition. Visualization and Mathematics, 3:113-134, 2003.

[13] J. Régis, J.F. Mangin, T. Ochiai, V. Frouin, D. Riviére, A. Cachia, M. Tamura, and Y. Samson. "Sulcal Root" Generic Model: a Hypothesis to Overcome the Variability of the Human Cortex Folding Patterns. Neurologia medico-chirurgica, 45(1):1-17, 2005.

[14] D.P. Richman, R.M. Stewart, J.W. Hutchinson, and V.S. Caviness Jr. Mechanical Model of Brain Convolutional Development. Science, 189(4196):18-21, 1975.

[15] R. Toro and Y. Burnod. A Morphogenetic Model for the Development of Cortical Convolutions. Cerebral Cortex, 15(12):1900-1913, 2005

[16] D.C. Van Essen. A tension-based theory of morphogenesis and compact wiring in the central nervous system. Nature, 385(6614):313-8, 1997

[17] H. Xue, L. Srinivasan, S. Jiang, M. Rutherford, AD Edwards, D. Rueckert, and JV Hajnal. Automatic cortical segmentation in the developing brain. Inf Process Med Imaging, 20:257-69, 2007.

[18] H. Xue, L. Srinivasan, S. Jiang, MA Rutherford, A.D. Edwards, D. Rueckert, and J.V. Hajnal. Longitudinal Cortical Registration for Developing Neonates. LECTURE NOTES IN COMPUTER SCIENCE, 4792:127, 2007.

Acknowledgements : This project is supported by... 